\title{
Introduction to the Special Issue in Honor of Joel Lebowitz
}

\author{
Michael Aizenman ${ }^{2}$ • Ivan Corwin ${ }^{1}$ • Jürg Fröhlich ${ }^{4}$ - Giovanni Gallavotti ${ }^{6}$. \\ Shelly Goldstein ${ }^{5} \cdot$ Herbert Spohn ${ }^{3}$
}

Published online: 10 July 2020

(c) Springer Science+Business Media, LLC, part of Springer Nature 2020

The following papers are dedicated to honoring Joel Lebowitz on the occasion of his ninetieth birthday and his retirement as editor-in-chief of the Journal of Statistical Physics (JSP). These contributions are written by a subset of his many friends, co-authors and admirers.

JSP was founded in 1969 by Howard Reiss, then a professor of chemistry at UCLA. Following the pioneering example of Lars Onsager, many chemists did research in statistical physics, rather than just in the more traditional field of thermodynamics. In the description of the board of editors of that time one reads: "Professor Joel L. Lebowitz, Statistical Mechanics of equilibrium and nonequilibrium, biomathematics, biophysics" - an impressive range of interests and competences.

In 1975, Joel was appointed editor-in-chief of JSP. Browsing through the volumes of this journal produced under Joel's leadership, one observes a substantial change in its scientific orientation. Joel was an active player and catalyser in an area of statistical physics that had emerged in the 60s and was now featuring spectacular progress: rigorous statistical mechanics. Papers published in JSP no longer came out of only physics or chemistry departments but were often written in mathematics departments. Besides statistical mechanics, Joel showed a very lively interest in several other fields, such as deterministic chaos, which then got featured in JSP. As one example, the late Mitchell Feigenbaum's seminal analysis of universality in the period-doubling bifurcations exhibited by families of maps from the unit interval to itself was published in JSP.

Joel made use of JSP to announce the programs of his legendary Statistical Mechanics Conferences. The first one to be announced there was number 34. It took place in December 1975 when Joel was still a professor at Yeshiva University. In December of this year the 123rd conference is scheduled to take place. In 1977, Joel moved to Rutgers University, where he holds the prestigious George William Hill Professorship of Mathematics and Physics.

The editorial board of JSP has traditionally been rather large, on the order of thirty members. It has been rejuvenated periodically. Its members have been tasked with attracting excellent papers to the journal and assisting in the reviewing of papers submitted for publication. Joel handled every single paper sent to the journal. Years ago, one would see stacks of brownish folders filled with papers considered for publication in JSP on his desk, in his car and at home. Nowadays, such stacks have become virtual-thanks to the 'Editorial Manager'. During his 43 years of service as editor-in-chief, over 10,000 papers submitted to JSP

Ivan Corwin

corwin@math.columbia.edu

Extended author information available on the last page of the article 
have gone through Joel's hands, an extraordinary accomplishment! Besides taking care of JSP, Joel has also edited, jointly with C. Domb, the series of review volumes entitled Phase Transitions and Critical Phenomena.

Joel has always had a brimming bag of open problems. In his scientific work he has emphasized the synergy between deep questions in science and mathematics. Mentoring and stimulating numerous students, postdoctoral researchers, and colleagues, his style has been to call attention to specific yet far reaching questions, formulated in the context of mathematical models. Forever fascinated with the origin of irreversibility in deterministic dynamics and with the meaning of entropy, Joel would often see far past technicalities, guiding collaborators in developing the details of an argument, all while keeping an eye on the essence which they reveal. Not surprisingly, he has co-authored hundreds of scientific papers spanning all aspects of statistical physics, many of which have become classics.

Beyond being a role model as a scientist and editor, Joel has been setting an example in his support of human rights, urging all to adopt it as both our individual and professional calling. He has had a life-long commitment to helping colleagues all over the world who were or are deprived of basic human rights and who are victims of persecution. The engagement of people like Joel is clearly crucial even if their efforts often take a long time to bear fruit.

We join David Ruelle, who said in his laudation when Joel was awarded the Henri Poincaré Prize: "I think we all recognise that Joel Lebowitz, as a scientist and as a man, has done a lot to make the world a better place to live. And for that we deeply thank him."

With great admiration, the co-editors of this special volume in Joel's honor:

Publisher's Note Springer Nature remains neutral with regard to jurisdictional claims in published maps and institutional affiliations.

\title{
Affiliations
}

Michael Aizenman ${ }^{2}$ - Ivan Corwin ${ }^{1}$ • Jürg Fröhlich ${ }^{4}$ - Giovanni Gallavotti ${ }^{6}$. Shelly Goldstein ${ }^{5} \cdot$ Herbert Spohn ${ }^{3}$

\author{
Michael Aizenman \\ aizenman@princeton.edu \\ Jürg Fröhlich \\ juerg@phys.ethz.c \\ Giovanni Gallavotti \\ giovanni.gallavotti@roma1.infn.it \\ Shelly Goldstein \\ oldstein@math.rutgers.edu \\ Herbert Spohn \\ spohn@ma.tum.de \\ 1 Department of Mathematics, Columbia University, Room 603, MC 4406, 2990 Broadway, New \\ York, NY 10027, USA \\ 2 Departments of Physics and Mathematics, Princeton University, Princeton, NJ 08544, USA \\ 3 Zentrum Mathematik and Physik Department, Technical University Munich, Boltzmannstr. 3, \\ 85747 Garching, Germany \\ 4 Prof. em. of Theoretical Physics, ETH Zurich, HIT K 42.3, Wolfgang-Pauli-Strasse 27, 8093 \\ Zurich, Switzerland
}


5 Rutgers University, 710 Hill Center, Piscataway, NJ 08854, USA

6 Dipartimento di Fisica, INFN, Universita' di Roma "La Sapienza”, P.le A. Moro 2, 00185 Rome, Italia 